\title{
BOUNDEDNESS PROPERTIES FOR SEMIGROUPS OF OPERATORS
}

\author{
FRODE TERKELSEN
}

\begin{abstract}
The note contains a characterization of an equicontinuity property for semigroups in metrizable spaces, and a result on essential boundedness for weakly measurable semigroups in metrizable locally convex spaces.
\end{abstract}

1. A set $\{T(\xi): \xi>0\}$ of continuous linear operators in a topological vector space is a semigroup, if $T(\xi+\eta)=T(\xi) T(\eta)$ for all $\xi, \eta>0$. In this note, boundedness properties for semigroups in metrizable spaces are investigated under weak assumptions. Proposition 1 states a local condition at the origin of $R$ which is equivalent with the existence of a set $S \subset(0, \infty)$ of measure zero such that $\{T(\xi): \xi \in[\alpha, \beta] \mid S\}$ is equicontinuous for any $[\alpha, \beta] \subset(0, \infty)$. No measurability or local convexity is assumed. Proposition 2 is concerned with weakly measurable semigroups in metrizable locally convex spaces. It is shown that certain sets of functions of the form $\xi \rightarrow\left\langle T(\xi) x, x^{\prime}\right\rangle$ are uniformly essentially bounded on compact subsets of $(0, \infty)$. As explained in $\S 3$, this note is closely related to results by W. Feller [1], and it is based on a connection between measure and topology stated in Lemma 1.

2. The word measurable means Lebesgue measurable throughout, $m$ denoting the Lebesgue measure on the real numbers $R$.

LeMma 1 (OstrowsKI). Let $K \subset R$ be a measurable set of positive measure, and let $D \subset R$ be dense in $R$. Then the set $R \backslash(D+K)$ is of measure zero.

Proof. We may assume that $D$ is countable, so that $A=D+K$ is measurable. Since $m(K)>0$, there exists a density point $\eta \in R$ of $K$. Given any $\xi \in R$, choose a sequence $\left(\sigma_{i}\right)$ in $D$ with $\lim _{i \rightarrow \infty} \sigma_{i}=\xi-\eta$. For any open interval $J$ containing 0 we have

$$
m\left(A \cap\left(\sigma_{i}+\eta+J\right)\right) \geqq m\left(\left(\sigma_{i}+K\right) \cap\left(\sigma_{i}+\eta+J\right)\right)
$$

for all $i$. In the limit we get $m(A \cap(\xi+J)) \geqq m(K \cap(\eta+J))$, implying that $\xi$ is a density point of $A$. Since $A$ is measurable, $m(R \backslash A)=0$ must hold.

Received by the editors October 31, 1972.

AMS (MOS) subject classifications (1970). Primary 47D05.

(c) American Mathematical Society 1973 
For concepts used in the sequel from the theory of topological vector spaces we refer to [5]. The scalar field is $R$ or the complex numbers.

LEMMA 2. Let $\{T(\xi): \xi>0\}$ be a semigroup of continuous linear operators in a metrizable topological vector space $E$. Then, for any $[\alpha, \beta] \subset(0, \infty)$ and any 0 -neighborhood $V$ in $E$, there exists a 0 -neighborhood $U$ in $E$ such that the set $\{\xi \in[\alpha, \beta]: T(\xi)(U) \subset V\}$ is dense in $[\alpha, \beta]$.

Proof. Let $\left\{U_{n}: n \in N\right\}$ be a 0 -neighborhood base in $E$ ( $N$ denoting the natural numbers). Define $P_{n}=\left\{\eta \in(0, \alpha): T(\eta)\left(U_{n}\right) \subset V\right\}$ for $n \in N$, then $(0, \alpha)=\bigcup_{n \in N} P_{n}$. By Baire's theorem there exists a $k \in N$ such that the closure of $P_{k}$ contains a nonempty open interval $J$. By compactness there is a finite set $Q \subset(0, \infty)$ with $[\alpha, \beta] \subset J+Q$, and then the set $D=[\alpha, \beta] \cap$ $\left(P_{k}+Q\right)$ is seen to be dense in $[\alpha, \beta]$. There exists a 0-neighborhood $U$ in $E$ such that $T(\sigma)(U) \subset U_{k}$ for each $\sigma \in Q$. For any $\xi \in D$, choose $\eta \in P_{k}$ and $\sigma \in Q$ with $\xi=\eta+\sigma$. Then $x \in U$ implies $T(\xi) x=T(\eta) T(\sigma) x \in T(\eta)\left(U_{k}\right) \subset$ $V$.

Proposition 1. Let $\{T(\xi): \xi>0\}$ be a semigroup of continuous linear operators in a metrizable topological vector space $E$. The following are equivalent:

(i) For any $\alpha>0$ and any 0 -neighborhood $V$ in $E$, there exist a measurable set $K \subset(0, \alpha)$ of positive measure and a 0 -neighborhood $U$ in $E$ such that $T(\xi)(U) \subset V$ for all $\xi \in K$.

(ii) There exists a set $S \subset(0, \infty)$ of measure zero such that for each $[\alpha, \beta] \subset(0, \infty)$, the set $\{T(\xi): \xi \in[\alpha, \beta] \backslash S\}$ is equicontinuous.

Proof. Clearly (ii) implies (i). Suppose (i) holds, and let $[\alpha, \beta] \subset(0, \infty)$ be given. Let $\gamma=\alpha / 2$, and let $\left\{U_{n}: n \in N\right\}$ be a 0 -neighborhood base. For each $n \in N$, choose a 0 -neighborhood $V_{n}$ according to Lemma 2 such that $D_{n}=\left\{\eta \in[\gamma, \beta]: T(\eta)\left(V_{n}\right) \subset U_{n}\right\}$ is dense in $[\gamma, \beta]$. By (i), choose a measurable set $K_{n} \subset(0, \gamma)$ with $m\left(K_{n}\right)>0$ and a 0 -neighborhood $W_{n}$ such that $T(\sigma)\left(W_{n}\right) \subset V_{n}$ for $\sigma \in K_{n}$. Define $S_{n}^{\prime}=[\alpha, \beta] \backslash\left(D_{n}+K_{n}\right)$, then $m\left(S_{n}^{\prime}\right)=0$ by Lemma 1 , observing that $[\alpha, \beta] \cap\left(\eta+K_{n}\right)$ is empty whenever $\eta \notin[\gamma, \beta]$. The set $S^{\prime}=\bigcup_{n \in N} S_{n}^{\prime}$ satisfies $m\left(S^{\prime}\right)=0$. Given any 0-neighborhood $V$, choose $n \in N$ such that $U_{n} \subset V$. For any $\xi \in[\alpha, \beta] \backslash S^{\prime}$, there exist $\eta \in D_{n}$ and $\sigma \in K_{n}$ with $\xi=\eta+\sigma$. Then $x \in W_{n}$ implies $T(\xi) x=T(\eta) T(\sigma) x \in$ $T(\eta)\left(V_{n}\right) \subset U_{n} \subset V$, showing that $\left\{T(\xi): \xi \in[\alpha, \beta] \mid S^{\prime}\right\}$ is equicontinuous. We conclude that for each $k \in N$ there exists $S_{k} \subset[1 / k, k]$ with $m\left(S_{k}\right)=0$ such that $\left\{T(\xi): \xi \in[1 / k, k] \mid S_{k}\right\}$ is equicontinuous. The set $S=\bigcup_{k \in N} S_{k}$ satisfies (ii).

A semigroup of operators in a locally convex space $E$ is said to be weakly measurable, if for each $\left(x, x^{\prime}\right) \in E \times E^{\prime}$ the function $\xi \rightarrow\left\langle T(\xi) x, x^{\prime}\right\rangle$ is measurable. 
Proposition 2. Let $\{T(\xi): \xi>0\}$ be a weakly measurable semigroup of continuous linear operators in a metrizable locally convex space $E$. Then, for any $[\alpha, \beta] \subset(0, \infty)$ and any equicontinuous set $H \subset E^{\prime}$, there exists a 0 -neighborhood $U$ in $E$ such that for each $\left(x, x^{\prime}\right) \in U \times H$ we have

$$
\left|\left\langle T(\xi) x, x^{\prime}\right\rangle\right| \leqq 1 \text { a.e. on }[\alpha, \beta] .
$$

Proof. The set

$$
U=\bigcap_{x^{\prime} \in H}\left\{x \in E:\left|\left\langle T(\xi) x, x^{\prime}\right\rangle\right| \leqq 1 \text { a.e. on }[\alpha, \beta]\right\}
$$

is easily seen to be convex and circled. We shall show that $U$ absorbs every bounded set in $E$, implying that $U$ is a 0 -neighborhood since $E$ is bornological. If a bounded set $B \subset E$ is not absorbed by $U$, then for each $n \in N$ there exists $x_{n} \in B$ such that $x_{n} \notin n U$. Hence, we get sequences $\left(x_{n}\right)$ in $B$ and $\left(x_{n}^{\prime}\right)$ in $H$ such that, for each $n \in N$,

$$
\left|\left\langle T(\xi) x_{n}, x_{n}^{\prime}\right\rangle\right|>n
$$

on a subset of $[\alpha, \beta]$ of positive measure. There exists a 0 -neighborhood $V$ such that $\left|\left\langle x, x^{\prime}\right\rangle\right| \leqq 1$ for $\left(x, x^{\prime}\right) \in V \times H$. Setting $\gamma=\alpha / 2$, there exist by Lemma 2 a 0-neighborhood $W$ and a countable set $D \subset[\gamma, \beta]$ dense in $[\gamma, \beta]$ with $T(\eta)(W) \subset V$ for $\eta \in D$. Define the extended real-valued function $f$ on $(0, \gamma)$ by

$$
f(\sigma)=\sup \left\{\left|\left\langle T(\eta+\sigma) x_{n}, x_{n}^{\prime}\right\rangle\right|:(\eta, n) \in D \times N\right\} .
$$

By the weak measurability, $f$ is measurable. Given $\sigma \in(0, \gamma)$, the set $T(\sigma)(B)$ is bounded, so there exists a $\lambda>0$ with $T(\sigma)(B) \subset \lambda W$. Then $(\eta, n) \in D \times N$ implies $T(\eta) T(\sigma) x_{n} \in T(\eta)(\lambda W) \subset \lambda V$, hence $\left|\left\langle T(\eta+\sigma) x_{n}, x_{n}^{\prime}\right\rangle\right| \leqq \lambda$. Therefore $f(\sigma) \leqq \lambda$, i.e. $f$ is real-valued. Choose a measurable set $K \subset(0, \gamma)$ with $m(K)>0$ and a $\mu \geqq 0$ such that $f(\sigma) \leqq \mu$ for $\sigma \in K$. The set $S=[\alpha, \beta] \backslash(D+K)$ is of measure zero by Lemma 1 . For each $\xi=\eta+\sigma \in D+K$ we have $\left|\left\langle T(\xi) x_{n}, x_{n}^{\prime}\right\rangle\right| \leqq f(\sigma) \leqq \mu$ for all $n \in N$, contradicting (1). We conclude that $U$ absorbs every bounded set in $E$.

3. Lemma 1 was proved by Ostrowski in [3], and a similar result was applied in semigroup theory by Feller [1]. The assumptions on $K$ in Lemma 1 may clearly be replaced by the assumption that $K$ is of positive inner measure. On the other hand, in Halmos [2, p. 70] an example is given of a set $E_{0}$ of positive outer measure and a dense set $B$ such that $R \backslash\left(B+E_{0}\right)$ fails to be of measure zero. Lemma 2 extends a result for Banach spaces by Feller [1].

Condition (i) of Proposition 1 is a local condition at the origin of $R$. This condition implies the continuity of the functions $\xi \rightarrow T(\xi) x$ for a weakly 
measurable and almost separably valued semigroup in any l.c. space [6]. It is an open problem whether condition (ii) of Proposition 1 implies the equicontinuity of $\{T(\xi): \xi \in[\alpha, \beta]\}$. For a semigroup in a metrizable l.c. space $E$, condition (ii) is easily proved to be equivalent with the following:

(a) For any $[\alpha, \beta] \subset(0, \infty)$ and any equicontinuous set $H \subset E^{\prime}$, there exists a 0 -neighborhood $U$ in $E$ such that

$$
\sup \left\{\left|\left\langle T(\xi) x, x^{\prime}\right\rangle\right|:\left(x, x^{\prime}\right) \in U \times H\right\} \leqq 1
$$

a.e. on $[\alpha, \beta]$.

The conclusion of Proposition 2 is generally strictly weaker than (a) above. Phillips [4] has constructed an example of a weakly measurable semigroup, in a Hilbert space, such that for each set $K \subset(0, \infty)$ with $m(K)>0$ the semigroup fails to be equicontinuous on $K$ (this property, which is slightly stronger than the property stated in [4], follows from a result of Ostrowski [3] on solutions to the equation $\psi(x+y)=\psi(x)+\psi(y))$. The assumptions of Proposition 2 are satisfied by the example, but (i)-(ii) of Proposition 1 and therefore (a) fail to hold. In this example we have $\left\langle T(\xi) x, x^{\prime}\right\rangle=0$ a.e. on $(0, \infty)$ for all $x$ and $x^{\prime}$.

Results by Feller [1, Theorem 2.2 and Lemma 2.6] imply that a weakly measurable semigroup in a Banach space $E$ satisfies:

(b) For any $[\alpha, \beta] \subset(0, \infty)$, there exists a $\mu \geqq 0$ such that for each $\left(x, x^{\prime}\right) \in E \times E^{\prime}$,

$$
\left|\left\langle T(\xi) x, x^{\prime}\right\rangle\right| \leqq \mu\|x\| \cdot\left\|x^{\prime}\right\| \text { a.e. on }[\alpha, \beta] .
$$

Proposition 2 is equivalent with (b) for semigroups in normed spaces and thus represents an extension of (b). Suppose the assumptions of Proposition 2 hold for a semigroup in a metrizable l.c. space $E$ (if, in addition, the semigroup is almost separably valued, then $\{T(\xi): \xi \in[\alpha, \beta]\}$ is equicontinuous for any $[\alpha, \beta] \subset(0, \infty),[6])$. The conclusion means that for each bounded set $B$ in $E$ and each equicontinuous set $H$ in $E^{\prime}$, the set of functions $\xi \rightarrow\left\langle T(\xi) x, x^{\prime}\right\rangle$, with $\left(x, x^{\prime}\right) \in B \times H$, is uniformly essentially bounded on any compact set $K \subset(0, \infty)$. Moreover, for each $x^{\prime} \in E^{\prime}$ one can define a continuous linear functional on $E$ by

$$
x \rightarrow \int_{K}\left\langle T(\xi) x, x^{\prime}\right\rangle d m .
$$

Let $F$ be the subspace of $E^{\prime}$ generated by all functionals of this type ( $F=\{0\}$ is possible), and let $\left\{T^{\prime}(\xi): \xi>0\right\}$ be the adjoint semigroup in $E^{\prime}$ defined by $\left\langle x, T^{\prime}(\xi) x^{\prime}\right\rangle=\left\langle T(\xi) x, x^{\prime}\right\rangle$. It can be shown that for each $x^{\prime} \in F$ the function $\xi \rightarrow T^{\prime}(\xi) x^{\prime}$ is continuous with respect to the strong topology $\beta\left(E^{\prime}, E\right)$ on $E^{\prime}$, partly extending [1, Theorem 3.3]. 


\section{REFERENCES}

1. W. Feller, Semi-groups of transformations in general weak topologies, Ann. of Math. (2) 57 (1953), 287-308. MR 14, 881.

2. P. R. Halmos, Measure theory, Van Nostrand, Princeton, N.J., 1950. MR 11, 504.

3. A. Ostrowski, Über die Funktionalgleichung der Exponentialfunktion und verwandte Funktionalgleichungen, Jber. Deutsch. Math.-Verein. 38 (1929), 54-62.

4. R. S. Phillips, On one-parameter semi-groups of linear transformations, Proc. Amer. Math. Soc. 2 (1951), 234-237. MR 12, 617.

5. H. H. Schaefer, Topological vector spaces, Macmillan, New York, 1966. MR 33 \#1689.

6. F. Terkelsen, On semigroups of operators in locally convex spaces, Proc. Amer. Math. Soc. 22 (1969), 340-343. MR 40 \#1813.

Institute of Economics, University of Copenhagen, Studiestraede 6, DK-1455 COPENhagen K, DenMark 